\title{
Experimental and Numerical Evaluation of Mechanical Properties of 3D-Printed Stainless Steel 316L Lattice Structures
}

\author{
M. Carraturo, G. Alaimo, S. Marconi, E. Negrello, E. Sgambitterra, C. Maletta, A. Reali, and F. Auricchio
}

Submitted: 31 December 2020 / Revised: 1 March 2021 / Accepted: 16 March 2021 / Published online: 12 April 2021

\begin{abstract}
Additive manufacturing (AM), and in particular selective laser melting (SLM) technology, allows to produce structural components made of lattice structures. These kinds of structures have received a lot of research attention over recent years due to their capacity to generate easy-to-manufacture and lightweight components with enhanced mechanical properties. Despite a large amount of work available in the literature, the prediction of the mechanical behavior of lattice structures is still an open issue for researchers. Numerical simulations can help to better understand the mechanical behavior of such a kind of structure without undergoing long and expensive experimental campaigns. In this work, we compare numerical and experimental results of a uniaxial tensile test for stainless steel 316L octet-truss lattice specimen. Numerical simulations are based on both the nominal as-designed geometry and the as-build geometry obtained through the analysis of $\mu$-CT images. We find that the use of the as-build geometry is fundamental for an accurate prediction of the mechanical behavior of lattice structures.
\end{abstract}

Keywords additive manufacturing, finite element analysis, micro-CT, selective laser melting, tensile test

\section{Introduction}

Selective laser melting (SLM) is an additive manufacturing (AM) technology belonging to the class of powder bed fusion machines, where a layer of metal powder is selectively melted by a laser beam (Ref 1). Such technology has gained increasing attention over the last decades due to its extreme design flexibility, short lead time, and the possibility to produce lightweight structures (Ref 2). However, these advantages come along with complex physical phenomena occurring in a highly localized area around the laser beam, making predictability and reproducibility of SLM parts still an open issue for even more widespread adoption of this technology (Ref 3).

In particular, SLM generates geometrical and microstructural defects which might severely affect the mechanical properties of the final part (Ref 4). Such process-induced defects become even more relevant in the case of production of small geometrical features (e.g., lattice structures), but the link

This invited article is part of a special topical focus in the Journal of Materials Engineering and Performance on Additive Manufacturing. The issue was organized by Dr. William Frazier, Pilgrim Consulting, LLC; Mr. Rick Russell, NASA; Dr. Yan Lu, NIST; Dr. Brandon D. Ribic, America Makes; and Caroline Vail, NSWC Carderock.

M. Carraturo, G. Alaimo, S. Marconi, E. Negrello, A. Reali, and F. Auricchio, Department of Civil Engineering and Architecture, University of Pavia, via Ferrata 3, 27100 Pavia, PV, Italy; and E. Sgambitterra and C. Maletta, Department of Mechanical Engineering, University of Calabria, Ponte P. Bucci 46 C, 87036 Rende, CS, Italy. Contact e-mail: alessandro.reali@unipv.it.

among process parameters, manufactured geometry, and mechanical properties of the part is still not completely understood (Ref 4). In the literature, we can find several works on the experimental characterization of mechanical properties of 3D-printed lattice structures (see, e.g., (Ref 5) and reference therein), whereas only a limited amount of works include numerical characterization, i.e., a virtual experiment based on finite element analysis (FEA).

Instead of an expensive and complex experimental characterization, numerical methods can be effectively and successfully employed to predict mechanical properties of SLM parts (Ref 6, 7). However, numerical characterization of SLM manufactured components is an extremely challenging task. Nowadays, numerical simulations are mostly used to check and eventually optimize process parameters to minimize processinduced defects in SLM components (Ref 8-10), whereas the use of FEA for the mechanical characterization of SLM parts is still burdened by the fact that the as-built geometry is substantially different from the as-designed original 3D virtual model generated within a computer-aided design (CAD) environment. Therefore, an accurate representation of the real, as-built geometry is needed to correctly predict the mechanical behavior of the structure by means of FEA.

The as-built geometry of AM parts can be directly extracted through an industrial-grade micro-computed tomography $(\mu-$ CT) able to reach a spatial resolution of a few microns. Moreover, $\mu$-CT data images can be employed to investigate material properties - such as density - and geometrical defects of the produced components. On one hand, running numerical analysis directly on $\mu$-CT images is quite a demanding task in terms of both memory consumption and computational power (Ref 11). On the other hand, the FEA of an AM part computed directly on the CAD model would lead to quite inaccurate results (Ref 12). Therefore, corrections (e.g., statistically equivalent models) are usually needed to include material and geometric defects within the digital twin of 3D-printed parts 


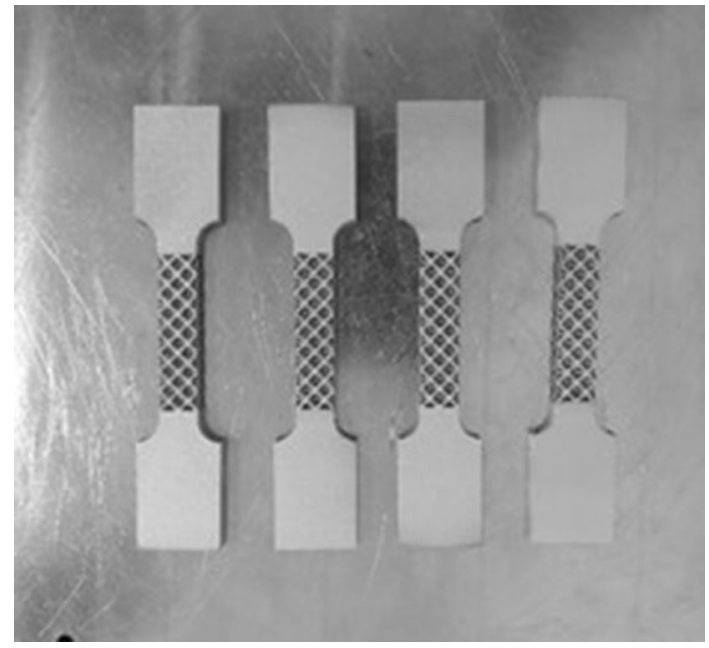

Fig. 1. 3D-printed lattice specimens

(Ref 13), or immersed boundary methods can be used to compute directly on $\mu-\mathrm{CT}$ images (Ref 14).

The objective of the present study is to investigate both experimentally and numerically the relationship between the process-induced defects present in the as-built component and its mechanical properties. Advantages, as well as limitations of numerical characterization of mechanical properties, are discussed in relation to the experimental evidence obtained for lattice tensile specimens produced by SLM.

The present article is organized as follows. Section 2 describes the experimental setup for an uniaxial tensile test of a lattice specimen. Section 3 introduces the numerical model together with the corresponding discretization used to simulate the uniaxial tensile test on the as-built geometry, obtained from $\mu$-CT images. Section 4 presents the main results, comparing numerical and experimental evidence and discussing some observations derived from the analysis of the $\mu$-CT data. Finally, in Section 5, we draw the main conclusions and possible further outlook of our research.

\section{Experimental Setup}

Four lattice specimens (Fig. 1) were printed with stainless steel 316L metal powder (SS316L-0447 Renishaw ${ }^{\mathrm{TM}}$ ) using the Renishaw $^{\mathrm{TM}}$ AM400 SLM system available at the 3DMetal@UniPV Laboratory of the University of Pavia. Table 1 reports the adopted process parameters. The as-designed lattice is composed of octet-truss cellular structures with a size of equal length of $6 \mathrm{~mm}$ and a density of $20 \%$, which leads to a truss thickness of $0.82 \mathrm{~mm}$, as depicted in Fig. 2. First, one lattice specimen geometry is acquired using a $\mu-\mathrm{CT}$ scan, to observe the geometrical and material defects present in the structure. The $\mu$-CT data acquisition on the tensile specimen was conducted with a Phoenix V|tome $\mid \mathrm{x}$ C (Waygate Technologies) machine and setting a voxel resolution of $32 \mu \mathrm{m}$

Secondly, two lattice specimens were tested on a uniaxial testing machine Instron E10000 under displacement control, adopting a displacement rate of $0.5 \mathrm{~mm} / \mathrm{min}$. Deformations were measured through contact extensometers as depicted in Fig. 3, whereas tensile tests were conducted up to a $4 \%$ strain
Table 1 Process parameters

\begin{tabular}{lr}
\hline Process parameters & \multicolumn{1}{c}{ Value } \\
\hline Build plate temperature & $170{ }^{\circ} \mathrm{C} \pm 1{ }^{\circ} \mathrm{C}$ \\
Chamber temperature & $35{ }^{\circ} \mathrm{C} \pm 5{ }^{\circ} \mathrm{C}$ \\
Layer thickness & $50 \mu \mathrm{m} \pm 1 \mu \mathrm{m}$ \\
Hatch spacing & $110 \mu \mathrm{m} \pm 2 \mu \mathrm{m}$ \\
Laser spot & $70 \mu \mathrm{m} \pm 1 \mu \mathrm{m}$ \\
Scan speed & $1200 \mathrm{~mm} / \mathrm{s} \pm 2 \mathrm{~mm} / \mathrm{s}$ \\
Laser power & $200 \mathrm{~W} \pm 0.1 \mathrm{~W}$ \\
\hline
\end{tabular}

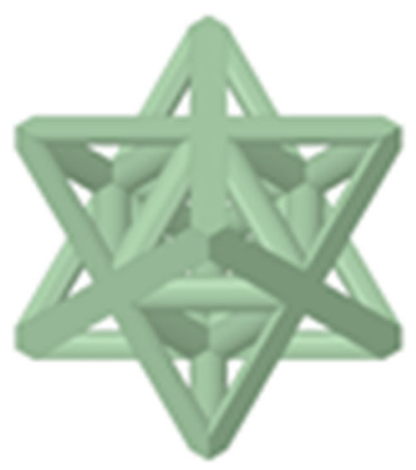

Fig. 2. CAD model of the Octet-truss cellular structure

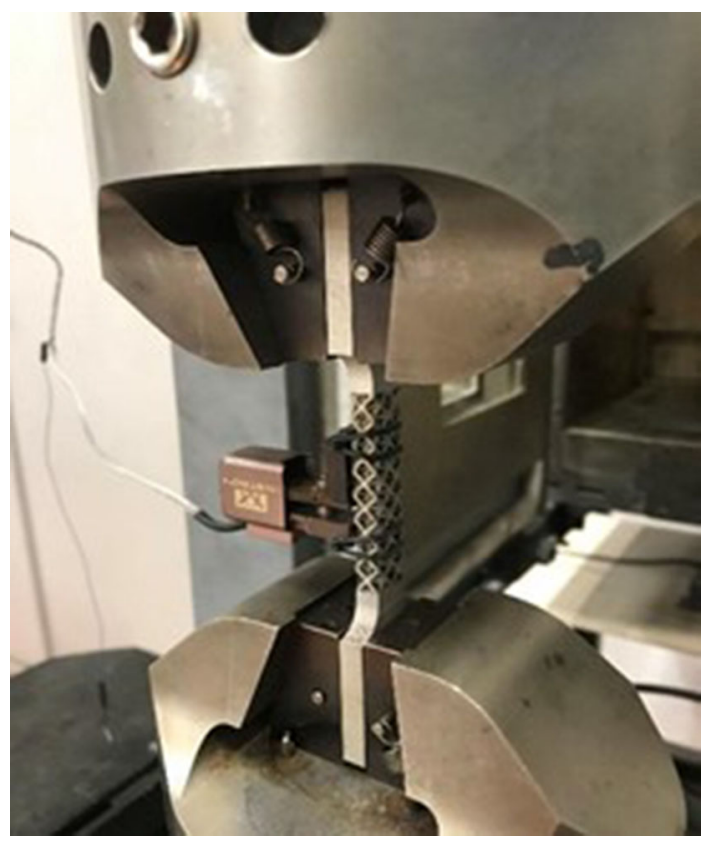

Fig. 3. Experimental setup of the uniaxial tensile test

value. The uniaxial tests were performed at the Mech-LAB Laboratory of the University of Calabria.

\section{Numerical Simulation Setup}

To simulate the tensile test behavior of the lattice specimen, we use Abaqus $\mathrm{CAE}^{\mathrm{TM}}$. Three different linear tetrahedral meshes are generated starting from the $\mu$-CT data (as-built 
geometry) setting different geometric tolerances equal to 75 , 50 , and $15 \mu \mathrm{m}$, respectively. Such a tolerance corresponds to the smallest geometrical features captured by the mesh. It is important to remark that the average dimension of a single powder particle is $30 \mu \mathrm{m}$, i.e., the finer mesh with a tolerance of $15 \mu \mathrm{m}$ is able to resolve the single powder particle (see Fig. 4). The corresponding number of elements and degrees of freedom (DOFs) of the resulting meshes are reported in Table 2. Moreover, we compute an additional simulation of the tensile test directly on the original CAD model (as-designed geometry) of the specimen. For FEA, we adopt an isotropic elastoplastic constitutive model with isotropic hardening, for which the parameters are the nominal values of stainless steel $316 \mathrm{~L}$ provided by Renishaw ${ }^{\mathrm{TM}}$ and reported in Table 3.

\section{Results and Discussion}

\subsection{Lattice Structure from $\mu$-CT}

Setting a threshold value of 40,000 Hounsfield units, we can evaluate the relative density of the as-built specimen equal to $21.3 \%$, thus slightly above the original, as-designed density of $20 \%$.

In Fig. 5, we can observe three different views of the specimen's $\mu$-CT image: the longitudinal, the transversal, and the top view, respectively. The top view shows a very regular printed surface, whereas, in the transversal and longitudinal views, we can observe several irregularities, due to hanging powder particles on surfaces opposite to the build direction. From the $\mu$-CT images of Fig. 5, we can also observe two kinds of pores within the structure: large irregularly shaped pores and small spherical pores. The former are on the boundary surfaces of the trusses and might lead to crack initiation and consequent propagation, whereas the latter are located within the structure and are much smaller and regularly shaped. According to (Ref 7 ), this second type of pores is associated with regions of lower density which originate from the partial remelting of surfaces of previous layers.

\subsection{Experimental and Numerical Tensile Behavior of 3D-Printed Octet-Truss Cellular Structures}

In Fig. 6 are reported the force-displacement curves of the numerical results together with the experimental data. The first observation is that the numerical results computed on the asdesigned geometry deliver strongly different values from the numerical results obtained on the as-built geometry and by means of experiments. The deviation between as-designed results and experimental values occurs already in the elastic regime, clearly showing a non-negligible influence of the as-

Table 2 Finite element discretization of the as-build lattice specimen

\begin{tabular}{lcc}
\hline Tolerance, $\boldsymbol{\mu m}$ & Number of elements & Number of DOFs \\
\hline 75 & $2,500,383$ & $1,712,370$ \\
50 & $3,025,050$ & $2,027,994$ \\
15 & $3,401,965$ & $2,257,851$ \\
\hline
\end{tabular}

Table 3 Material parameters of the numerical model to be used in Abaqus

\begin{tabular}{ll}
\hline Material parameter & \multicolumn{1}{c}{ Value } \\
\hline Young's modulus & $190[\mathrm{GPa}]$ \\
Poisson ratio & $0.27[-]$ \\
Plastic strain & $0.067446[-]$ \\
Yield stress & $428[\mathrm{MPa}]$ \\
\hline
\end{tabular}

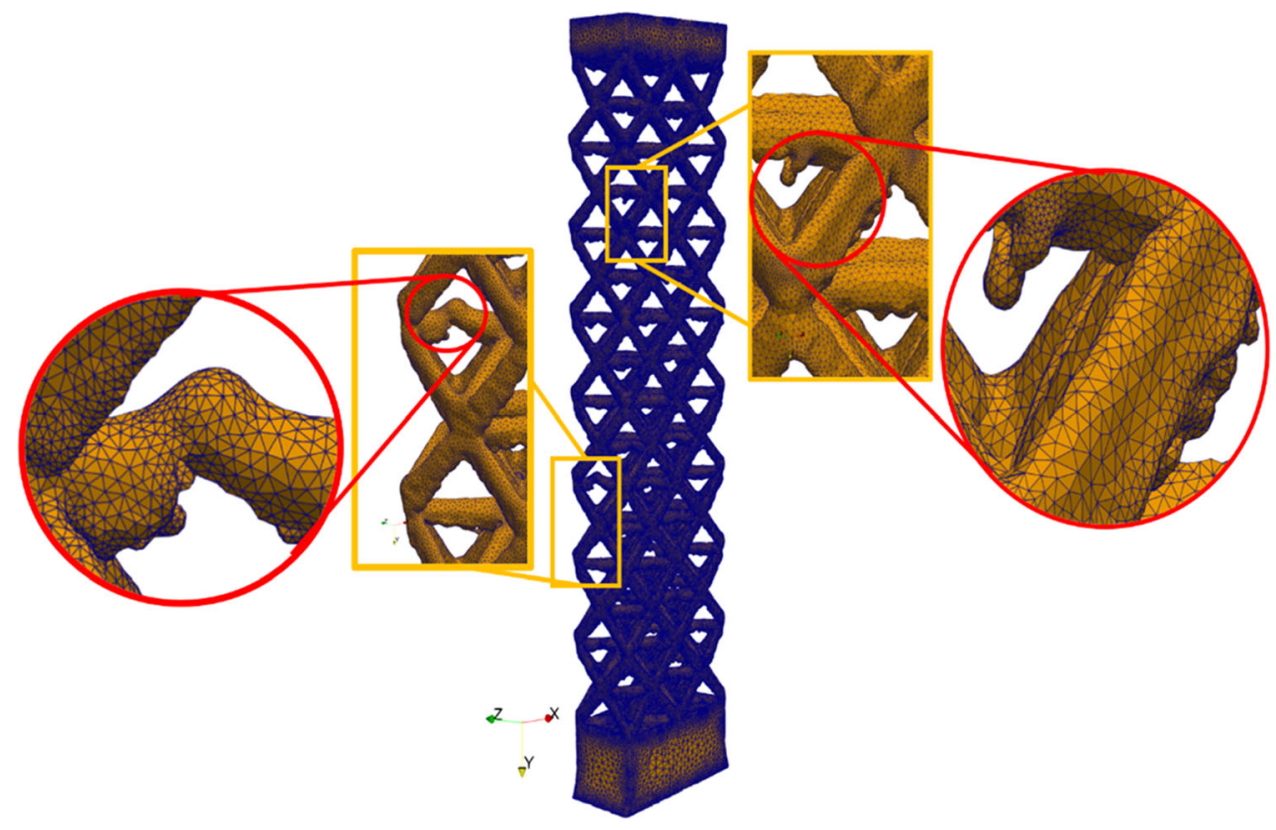

Fig. 4. Finite element discretization of the as-built geometry obtained from $\mu$-CT using a tolerance of $15 \mu \mathrm{m}$. The element edges are in blue in the figure 

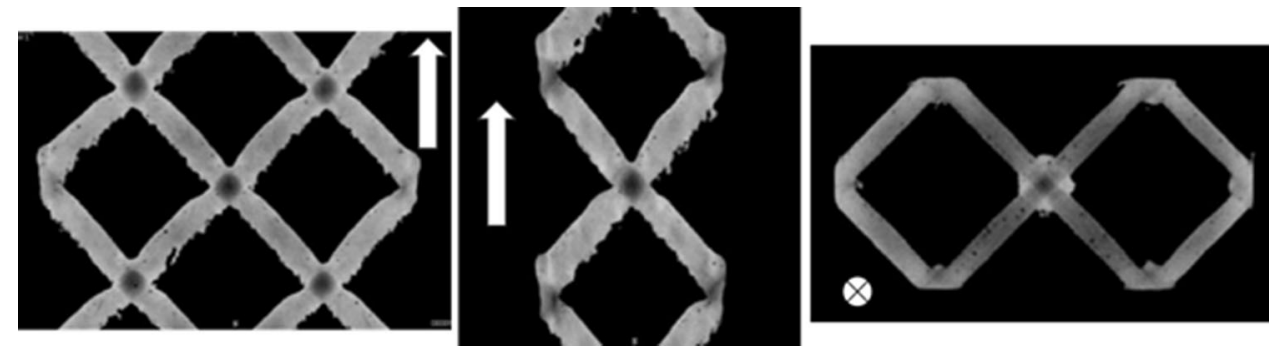

Fig. 5. $\mu$-CT-scan image of a portion of the lattice specimen. From left to right, we have the longitudinal, the transversal, and the top view of the $\mu$-CT-scan image. The white arrow indicates the build direction

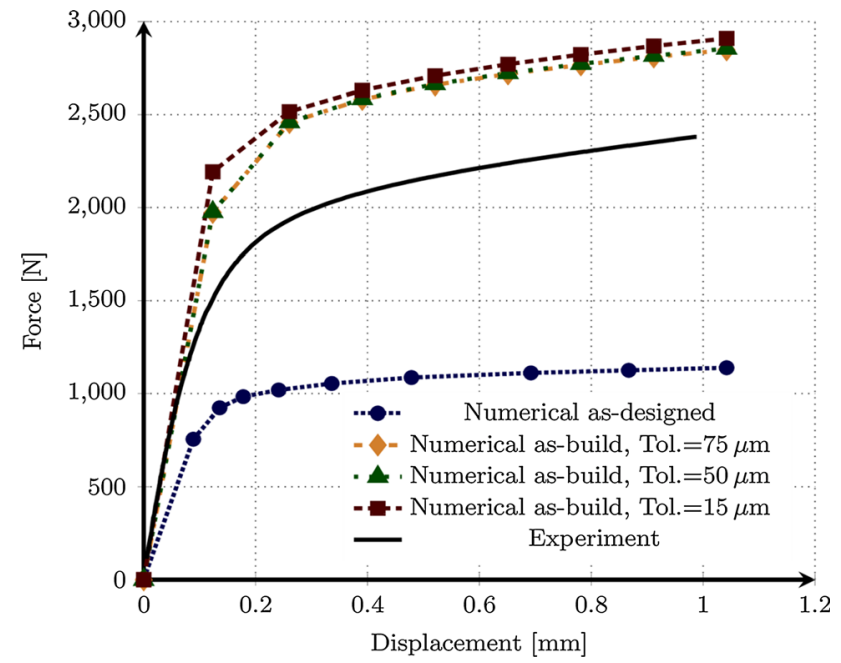

Fig. 6. Force-displacement curves from the uniaxial tensile test. Experimental curve is plot together with the numerical curves obtained from both as-designed geometry and as-built geometry with different mesh tolerances. We can observe that both numerical curves present a non-negligible deviation from the experimental curve, but the numerical curves using as-built geometry better approximate the experimental results compared to the numerical asdesigned curve

built geometry on the accuracy of the numerical simulation. This observation is in line with what reported in the literature (Ref 15, 16). Moreover, as-built geometry leads to numerical results closer to experimental values compared to the results obtained using the as-designed geometry in both elastic and plastic regimes and with a small influence of the chosen mesh tolerance. Such a small difference among results of different discretizations highlights the negligible influence of extremely small features on the overall mechanical behavior of the lattice structures, which is instead mainly affected by the macroscopic deviation occurring between the as-design and the as-built geometry correctly captured by all the chosen mesh tolerances. However, as-built results present a non-negligible error with respect to experimental measurements in the plastic region (approximately $21 \%$ relative error at $1 \mathrm{~mm}$ displacement). In particular, we observe that the experimental curve starts yielding at lower values compared to the simulated as-built geometry curve. From the $\mu$-CT images analysis conducted in Section 4.1, we have observed two kinds of pores: large pores on the surface which are generally correctly captured by the mesh, and small internal pores which are instead neglected in the numerical analysis where we adopt a homogeneous material model with constant properties. Therefore, we can reasonably guess that these small pores - due to partial remelting of the material - contribute to lower the value of the force-displacement curve as we observe from experiments, but more results are needed to confirm such an observation.

\section{Conclusions}

In the present work, by means of SLM technology we design and produce two octet-truss lattice tensile specimens. Uniaxial tensile tests are conducted on the lattice specimens both experimentally and numerically. The numerical simulations are performed adopting the bulk elastoplastic material properties and using two different geometries: the as-built geometry obtained from $\mu$-CT data images and the as-designed geometry from the $3 \mathrm{D}$ CAD model. It is observed that FEA using the as-designed geometry fails to replicate experimental data, whereas numerical results based on as-built geometry are closer to the experimental measurements. Nevertheless, a still not negligible error occurs between as-built numerical and experimental results, possibly due to the non-homogeneous material distribution within lattice trusses that we can observe from $\mu$-CT images. The further outlook of the present work aims at investigating more in detail the origin of this discrepancy among FEA based on the as-built geometry and experimental data.

\section{Acknowledgments}

This work was partially supported by the Italian Minister of University and Research through the MIUR-PRIN projects "A BRIDGE TO THE FUTURE: Computational methods, innovative applications, experimental validations of new materials and technologies" (No. 2017L7X3CS) and "XFAST-SIMS“" (No. $20173 \mathrm{C} 478$ N). The authors gratefully acknowledge Giorgio Vattasso from LaborMet Due (http://www.labormetdue.it/) for his technical support in obtaining CT scan images.

\section{Funding}

Open access funding provided by Università degli Studi di Pavia within the CRUI-CARE Agreement. 


\section{Open Access}

This article is licensed under a Creative Commons Attribution 4.0 International License, which permits use, sharing, adaptation, distribution and reproduction in any medium or format, as long as you give appropriate credit to the original author(s) and the source, provide a link to the Creative Commons licence, and indicate if changes were made. The images or other third party material in this article are included in the article's Creative Commons licence, unless indicated otherwise in a credit line to the material. If material is not included in the article's Creative Commons licence and your intended use is not permitted by statutory regulation or exceeds the permitted use, you will need to obtain permission directly from the copyright holder. To view a copy of this licence, visit http://creativecommons.org/licenses/by/4.0/.

\section{References}

1. ASTM International, ASTM Standard F2792. Standard Terminology for Additive Manufacturing Technologies, West Conshohocken, 2012

2. I. Gibson, D. Rosene, and B. Stucker, Additive Manufacturing Technologies, Springer, New York, 2010

3. J.L. Bartlette, and X. Li, An Overview of Residual Stresses in Metal Powder Bed Fusion, Add. Manuf., 2019, 27, p 131-149

4. H. Yeung, B. Lane, M. Donmez, J. Foxe, and J. Neira, Implementation of Advanced Laser Control Strategies for Powder Bed Fusion Systems, Proc. Manuf., 2018, 26, p 871-879

5. H. Yeung, B. Lanee, and J. Fox, Part Geometry and Conduction-Based Laser Power Control for Powder Bed Fusion Additive Manufacturing, Add. Manuf., 2019, 30, p 100844

6. D. Herzog, V. Seyda, E. Wyciske, and C. Emmelmann, Additive Manufacturing of Metals, Acta Mater., 2016, 117, p 371-392

7. O. Al-Ketan, R. Rowshane, and R.K. Abu Al-Rub, TopologyMechanical Property Relationship of 3D Printed Struts, Skeletal, and
Sheet Based Periodic Metallic Cellular Materials, Add. Manuf., 2018, 19, p 167-183

8. C. Qiu, N. Adkinse, and M. Attalleh, Microstructure and Tensile Properties of Selectively Laser-Melted and of HIPed Laser-Melted Ti6Al-4V, Mater. Sci. Eng. A, 2013, 578, p 230-239

9. R. Ganeriwala, M. Strantza, W. King, B. Clausen, T. Phan, L. Levine, D. Browne, and N. Hodge, Evaluation of a Thermomechanical Model for Prediction of Residual Stress During Laser Powder Bed Fusion of Ti-6Al-4V, Add. Manuf., 2019, 27, p 489-502

10. M. Carraturo, J. Jomo, S. Kollmannsberger, A. Reali, F. Auricchio, and R. Ernst, Modeling and Experimental Validation of an Immersed Thermo-Mechanical Part-Scale Analysis for Laser Powder Bed Fusion Processes, Add. Manuf., 2020, 36, p 101498

11. L. Parry, I. Ashcroft, and R. Wildman, Geometrical Effects on Residual Stress in Selective Laser Melting, Add. Manuf., 2019, 25, p 166-175

12. N. Kurshunova, J. Jomo, G. Lékó, D. Reznik, P. Balázs, and S. Kollmannsberger, Image-Based Material Characterization of Complex Microarchitectured Additively Manufactured Structures, Comput. Math. Appl., 2020, 80, p 2462-2480

13. M. Kästner, U. Gebhardt, J.K.K.U. Hufenbach, M. Berner, and S. Holtzhausen, Virtual Testing on Geometricallz Imperfect Additivelz Manufactured Lattice Structures, II International Conference on Simulation for Additive Manufactuing (Sim_AM19), Pavia, 2019

14. S. Arabnejad, and D. Pasini, Mechanical Properties of Lattice Materials Via Asymptotic Homogenization and Comparison with Alternative Homogenization Methods, Int. J. Mech. Sci., 2013, 77, p 249-262

15. N. Korshunova, G. Alaimo, S. Hosseini, M. Carraturo, A. Reali, J. Niiranen, F. Auricchio, E. Rank, and S. Kollmannsberger, Image-Based Numerical Characterization of Tensile Behavior of Octet-Truss Structures and its Experimental Validation, Add. Manuf.

16. L. Liu, P. Kamm, F. García-Moreno, J. Banhart, and D. Pasini, Elastic and Failure Response of Imperfect Three-Dimensional Metallic Lattices: The Role of Geometric Defects Induced by Selective Laser Melting, J. Mech. Phys. Solids, 2017, 107, p 160-184

Publisher's Note Springer Nature remains neutral with regard to jurisdictional claims in published maps and institutional affiliations. 